\title{
Paradox and Logical Revision. A Short Introduction
}

\author{
Julien Murzi $\cdot$ Massimiliano Carrara
}

Published online: 14 December 2014

(C) Springer Science+Business Media Dordrecht 2014

Logical orthodoxy has it that classical first-order logic, or some extension thereof, provides the right extension of the logical consequence relation. However, together with naïve but intuitive principles about semantic notions such as truth, denotation, satisfaction, and possibly validity and other naïve logical properties, classical logic quickly leads to inconsistency, and indeed triviality. At least since the publication of Kripke's Outline of a theory of truth (Kripke 1975), an increasingly popular diagnosis has been to restore consistency, or at least non-triviality, by restricting some classical rules. Our modest aim in this note is to briefly introduce the main strands of the current debate on paradox and logical revision, and point to some of the potential challenges revisionary approaches might face, with reference to the nine contributions to the present volume. $^{1}$

Our discussion is structured thus. Section 1 reviews the Liar and the Knower paradoxes. Section 2 briefly discusses four revisionary approaches. Section 3 sketches a potential challenge for revisionary approaches to semantic paradox. For reasons of space, we have mostly aimed at presenting

J. Murzi ( $\square)$

University of Kent and Munich Center for Mathematical Philosophy, Ludwig-Maximilians Universität, Munich, Germany e-mail: j.murzi@gmail.com

\section{Carrara}

FISPPA Department-Section of Philosophy, University of Padua, Padua, Italy

e-mail: massimiliano.carrara@unipd.it

M. Carrara

Cogito-Research center of Philosophy, Bologna, Italy the big picture, in broad strokes, thus sacrificing many important details.

\section{Liars \& Co}

Begin with the assumption that truth naïvely plays capture and release (Beall 2007a, 2009), in the following minimal sense:

$$
{ }_{T r-\mathrm{I}} \frac{\Gamma \vdash \phi}{\Gamma \vdash \operatorname{Tr}(\ulcorner\phi\urcorner)} \quad \operatorname{Tr} \mathrm{- \textrm {E }} \frac{\Gamma \vdash \operatorname{Tr}(\ulcorner\phi\urcorner)}{\Gamma \vdash \phi},
$$

where $\operatorname{Tr}(x)$ expresses truth and $\ulcorner\phi\urcorner$ is a name of $\phi$. Somewhat less minimally, $\operatorname{Tr}(x)$ may be assumed to further satisfy the T-Scheme

$$
\text { (T-Scheme) } \operatorname{Tr}(\ulcorner\phi\urcorner) \leftrightarrow \phi,
$$

or, even less minimally, transparency: that $\operatorname{Tr}(\ulcorner\phi\urcorner)$ and $\phi$ are always intersubstitutable salva veritate in all non-opaque contexts. Next, assume that our language contains a sentence $\lambda$ identical to $\neg \operatorname{Tr}(\ulcorner\lambda\urcorner)$, so that $\lambda$ says of itself that it isn't true. Finally, let us further assume that the standard structural rules-rules in which no logical expression essentially figure, governing the structure of the consequence relation-are in place:

\footnotetext{
${ }^{1}$ For a recent introduction to non-classical theories of truth and other semantic notions, see the excellent Beall and Ripley (2014).
} 


$$
\begin{gathered}
\text { (Id) } \frac{\text { (SContr) } \frac{\Gamma, \phi, \phi \vdash \psi}{\Gamma, \phi \vdash \psi}}{\phi \vdash \phi} \\
\text { (Cut) } \frac{\Gamma \vdash \phi \quad \Delta, \phi \vdash \psi}{\Gamma, \Delta \vdash \psi},
\end{gathered}
$$

and that negation satisfies its standard I- and E-rules:

$$
\frac{\Gamma, \phi \vdash \perp}{\Gamma \vdash \neg \phi} \neg-\mathrm{I} \quad \frac{\Gamma \vdash \phi \quad \Delta \vdash \neg \phi}{\Gamma, \Delta \vdash \perp} \neg-\mathrm{E},
$$

where $\perp$ is a falsum constant. We may then reason thus. Let $\Pi$ be the following derivation of the theorem $\neg \operatorname{Tr}(\ulcorner\lambda\urcorner)$ :

$$
\begin{aligned}
& \frac{\operatorname{Tr}(\ulcorner\lambda\urcorner) \vdash \operatorname{Tr}(\ulcorner\lambda\urcorner)}{\operatorname{Tr}(\ulcorner\lambda\urcorner) \vdash \lambda} \operatorname{Tr} \text { - } \\
& \frac{\overline{\operatorname{Tr}(\ulcorner\lambda\urcorner) \vdash \neg \operatorname{Tr}\ulcorner\lambda\urcorner} \quad \operatorname{Tr}(\ulcorner\lambda\urcorner) \vdash \operatorname{Tr}(\ulcorner\lambda\urcorner)}{\frac{\operatorname{Tr}(\ulcorner\lambda\urcorner), \operatorname{Tr}(\ulcorner\lambda\urcorner) \vdash \perp}{\frac{\operatorname{Tr}(\ulcorner\lambda\urcorner) \vdash \perp}{\vdash \neg \operatorname{Tr}(\ulcorner\lambda\urcorner)} \neg-\mathrm{I}} \text { scontr }}
\end{aligned}
$$

Using $\Pi$, we can then 'prove' $\operatorname{Tr}(\ulcorner\lambda\urcorner)$ :

$$
\begin{aligned}
& \text { II } \\
& \frac{\vdash \neg \operatorname{Tr}(\ulcorner\lambda\urcorner)}{\frac{\vdash \lambda}{\vdash \operatorname{Tr}(\ulcorner\lambda\urcorner)}}
\end{aligned}
$$

This is the Liar Paradox.

The paradox can be strengthened using a predicate $\square(x)$ satisfying the rule of necessitation and the predicate equivalent of the $T$ axiom in modal logic:

$$
\text { (NEC) } \frac{\vdash \phi}{\vdash \square(\ulcorner\phi\urcorner)} \quad \text { (FACT) } \vdash \square(\ulcorner\phi\urcorner) \rightarrow \phi .
$$

To do so, it is sufficient to define a sentence $\kappa$ identical to $\neg \square(\ulcorner\kappa\urcorner)$, interpret $\operatorname{Tr}(x)$ as $\square(x)$ in the above derivation, and replace uses of $T r$-I and $T r$-E with, respectively, uses of NEC and FACT. The resulting reasoning, a 'proof' of $\square(\ulcorner\kappa\urcorner)$ and $\neg \square(\ulcorner\kappa\urcorner)$, is known as the Knower Paradox (Kaplan and Montague 1960; Myhill 1960). $\square(x)$ may be interpreted in a number of ways: some epistemic, such as knowledge or informal provability; some non-epistemic, such as validity and necessity. ${ }^{2}$

Notice the general form of the foregoing paradoxical arguments:

\footnotetext{
${ }^{2}$ We will further consider in Sect. 3 paradox-prone non-epistemic predicates such as determinate and stable truth.
}

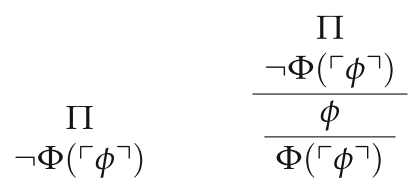

One establishes $\neg \Phi(\ulcorner\phi\urcorner)$ by means of an argument $\Pi$, here involving a release principle ( $\operatorname{Tr}$-E or FACT), ᄀ-I, $\neg$-E, and SContr. Then, the definition of $\phi$ and a capture principles such as $\operatorname{Tr}$-I and NEC allow one to conclude $\phi$, whence $\Phi(\ulcorner\phi\urcorner)$. This striking similarity strongly suggests that the Liar and the Knower paradoxes are little more than notational variants of each other. Notice, too, that $\Pi$ need not involve $\neg$-I, $\neg$-E, SContr, and Cut; as we'll see in Sect. 3, there may be other ways to establish $\neg \Phi(\ulcorner\phi\urcorner)$. Finally, it is worth pointing out that the above paradoxical reasonings also presuppose the validity of Cut since, in a natural deduction format, ᄀ-E effectively codifies a restricted form of transitivity, as the following derivation shows:

$$
\frac{\Gamma \vdash \phi \quad \frac{\Delta, \phi \vdash \perp}{\Delta \vdash \neg \phi}-\text {-I }}{\Gamma, \Delta \vdash \perp} \text {-E. }
$$

The natural way of establishing the premises of an application of $\neg$-E is but an instance of Cut.

It would seem, then, that notions such as truth, necessity, knowledge, validity, and informal provability are all provably inconsistent-indeed trivial, if the logic validates the principle of ex contraditione quodlibet, that a contradiction entails any sentence whatsoever:

$(\mathrm{ECQ}) \perp \vdash \phi$.

If it is thought that naïve semantic principles are, for some reason or other, non-negotiable, then one must blame the logic in order to restore consistency, or at least non-triviality. To be sure, such a revision is not to be taken lightly (see Terzian, THIS volumE), and there is no shortage of classical treatments, either hierarchical (Tarski 1936; Parsons 1974b; Burge 1979; Williamson 1998; Glanzberg 2001, 2004a; Schurz 2011), or non-hierarchical (Kripke 1975; McGee 1991; Gupta and Belnap 1993; Maudlin 2004; Leitgeb 2005; Simmons 1993, 2000, тнIS volumE). But, it has been argued, the alternatives are dire (Kripke 1975; Field 2008), the naïve semantic principles are non-negotiable (Field 2008; Beall 2009; for a criticism of their arguments, see Zardini, THIS vOLUME), and there might be independent reasons for putting the blame on the logic in the first place (Ripley, THIS volume; Zardini, THIS volume). So how can logic be revised on the face of semantic paradox? 


\section{Four Revisionary Approaches}

Each of $\neg-\mathrm{I}, \neg$-E, and SContr, and Cut can, and indeed has been, questioned. We very briefly consider the corresponding four revisionary strategies in turn.

\subsection{Paracomplete and Paraconsistent}

The most popular revisionary approaches to the Liar and Knower paradoxes involve revising the classical (intuitionistic) theory of negation, according to which $\neg$ satisfies both $\neg$-I and $\neg$-E. Thus, so-called paracomplete theorists hold that 'paradoxical' sentences such as $\lambda$ and $\kappa$ are gappy, in the sense of lacking a truthvalue, or having an intermediate value in between truth and falsity. Hence, negation fails to be exhaustive, i.e. it fails to satisfy the Law of Excluded Middle:

(LEM) $\vdash \phi \vee \neg \phi .{ }^{3}$

Moreover, $\neg$-I can no longer hold in general either: if $\phi$ is gappy, the fact that it entails $\perp$ does not yet show that it is false. Dually, paraconsistent logicians treat the Liar Paradox as a proof of $\operatorname{Tr}(\ulcorner\lambda\urcorner)$ and $\neg \operatorname{Tr}(\ulcorner\lambda\urcorner)$. In their view, negation fails to be exclusive: there is an overlap between truth and falsity, i.e. 'paradoxical' sentences are glutty, and ECQ must be given up. ${ }^{4}$ But, unless a new conditional is added to the language (about which more in a moment), $\neg$-E, and indeed $\rightarrow-\mathrm{E}$ (modus ponens) must be given up too: these rules fail to preserve truth for any $\phi$ that is both true and false (Priest 2006b; Beall 2009).

Kripke (1975) famously showed how to construct models for languages in which the truth-predicate is fully transparent, provided LEM and $\neg$-I (among other rules) are suitably restricted. More recently, Field $(2006,2008)$ and Brady (2006) have both defined models for paracomplete theories containing a conditional $\rightarrow$ satisfying $\vdash \phi \rightarrow \phi$, $\rightarrow-E$, and all instances of the T-Scheme. Because of Curry's Paradox, the conditional does not, and cannot, satisfy $\rightarrow-$ I. ${ }^{5}$ Similar (dual) results hold for paraconsistent languages (Priest 2006b; Beall 2009).

Defining a 'suitable' conditional strong enough to sustain ordinary reasoning and weak enough to avoid Curry's Paradox has proved to be no trivial enterprise. ${ }^{6}$ Some are

\footnotetext{
3 See e.g. Kripke (1975), Brady (2006), Soames (1999), Field (2008), Horsten (2009).

${ }^{4}$ See e.g. Asenjo (1966), Asenjo and Tamburino (1975), Priest (1979), Goodship (1996) Beall (2009), Beall (2011).

5 The standard conditional-involving Curry Paradox involves a sentence $\gamma$ identical to $\operatorname{Tr}(\ulcorner\gamma\urcorner) \rightarrow \perp$. Given the standard structural rules, $\rightarrow$-I, and $\rightarrow$-E, a Liar-like argument allows one to 'prove' $\perp$.

6 The conditional in question would need to serve as a means to express restricted quantification, as in 'Everyone in the room is happy'. It can be shown, however, that a 'suitable' conditional weak enough not to trigger Curry's Paradox is bound not to validate certain
}

more optimistic (Field 2013); others less so (Martin 2011). Partly for this reason, Beall $(2011,2014 b$, a) has recently advocated, following Goodship (1996), a detachment-free glut-theoretic approach to paradox-one that gives up the project of defining a 'suitable' conditional, and decidedly embraces the failure of $\rightarrow$-E displayed by basic paraconsistent logics. Horsten (2009) essentially advocates a dual strategy in a paraconsistent setting.

But does revising the logic of $\neg, \rightarrow$, and more generally operational rules, i.e. rules specifically governing the use of logical operators, suffice to solve the semantic paradoxes in general? The paradoxes of naïve logical properties suggest a negative answer to this question (Beall and Murzi 2013; Zardini 2013a, 2014a).

\subsection{Paradoxes of Naïve Logical Properties}

Consider the two following principles: that if $\psi$ is a consequence of $\phi$, then the argument $\langle\phi \therefore \psi\rangle$ is valid (henceforth, VP), and that one may conditionally assert $\psi$ given the assumptions that $\phi$ and that the argument $\langle\phi \therefore \psi\rangle$ is valid (henceforth, VD). More formally:

$$
\text { (VP) } \frac{\phi \vdash \psi}{\varnothing \vdash \operatorname{Val}(\ulcorner\phi\urcorner,\ulcorner\psi\urcorner)}(\mathrm{VD}) \frac{\Gamma \vdash \operatorname{Val}(\ulcorner\phi\urcorner,\ulcorner\psi\urcorner) \quad \Delta \vdash \phi}{\Gamma, \Delta \vdash \psi},
$$

where $\operatorname{Val}(x, y)$ expresses validity. Now let $\pi$ be a sentence identical to $\operatorname{Val}(\ulcorner\pi\urcorner,\ulcorner\perp\urcorner)$, so that $\pi$ says of itself that it validly entails absurdity. Then, courtesy of VD, one can easily derive $\perp$ from two occurrences of $\pi$ and conclude $\operatorname{Val}(\ulcorner\pi\urcorner,\ulcorner\perp\urcorner)$ by discharging both occurrences via a single application of VP. But, then, $\perp$ follows on no assumptions via VD. This is the Validity Curry Paradox, or v-Curry, for short (Whittle 2004; Shapiro 2011; Beall and Murzi 2013).

Beyond VP and VD, the argument only appeals to the standardly accepted structural rules. The validity of SContr in the above informal reasoning is presupposed by the multiple discharge of $\pi$ (Negri and von Plato 2001). As for Cut, it is effectively built in our formulation of VD. At a glance, the argument can be presented thus. Let $\Sigma$ be the following derivation of the theorem $\operatorname{Val}(\ulcorner\pi\urcorner,\ulcorner\perp\urcorner)$ :

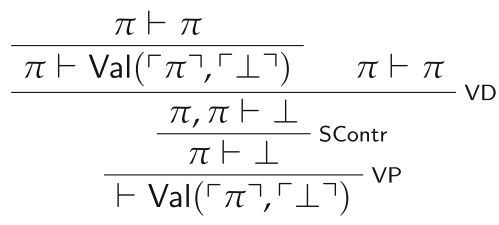

Footnote 6 continued

very intuitive principles about restricted quantification see e.g. Beall et al. (2006), Field (2013), Ripley (2014), Zardini (2014c). 
Using $\Sigma$, we can then 'prove' $\perp$ :

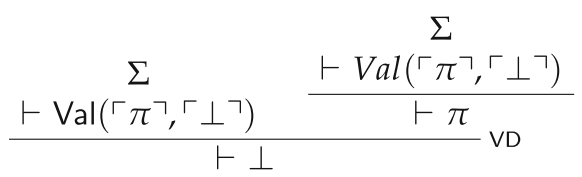

If paradoxes are to be solved via logical revision, a naïve conception of validity forces the rejection of one of the standardly accepted structural rules (Beall and Murzi 2013). Similar paradoxes plague other naïve logical properties, such as consistency and compatibility (Zardini 2013c).

The foregoing paradoxes leave revisionary theorists with two choices. Either blame the naïve semantic principles (see e.g. Ketland 2012; Cook 2013; Zardini 2013c; Field 2014), or restrict one of the structural rules (Shapiro 2011; Beall and Murzi 2013; Zardini 2011; Murzi 2012; Zardini 2014a). Concerning the first possibility, VP and VD may be called into question, respectively, by paracomplete theorists who already reject $\rightarrow-$ I and by paraconsistent theorists who already reject $\rightarrow$-E. What is more, Zardini (2013c) and Field (2014) have recently objected that, on the assumption that the validity relation is recursively enumerable, VD contradicts Gödel's Second Incompleteness Theorem.

We briefly mention some possible substructuralist rejoinders. First, it is unclear whether the natural paracomplete and paraconsistent arguments against VP and VD carry over to the paradoxes of consistency and, especially, compatibility. Second, it is hard to see why one should not be in a position to assert that the argument $\langle\phi \cdot: \psi\rangle$ is valid if presented with a derivation of $\psi$ from $\phi$ (see Shapiro, 2010 and Priest, THIS VOLUME). Third, even conceding that VP may be problematic, it is possible to derive a version of the v-Curry Paradox from VD alone and the plausible claim that $\phi \vdash \psi$ iff 'the argument $\langle\phi \cdot: \psi\rangle$ is valid' is true (Shapiro 2013). Fourth, Gödel's First Incompleteness Theorem suggests that validity outstrips derivability in any recursively enumerable system to which Gödel's incompleteness theorems apply, and hence may not be recursively enumerable (Myhill 1960; Murzi 2014a, c). Let us consider, then, the second choice, viz. to restrict one of the standardly accepted structural rules.

\subsection{Substructural Approaches: SContr-free and Cut- free}

Substructural approaches are usually met with skepticism. Thus, Field writes:

I haven't seen sufficient reason to explore this kind of approach (which I find very hard to get my head around), since I believe we can do quite well without it. (Field 2008, pp. 10-11)

While the paradoxes of naïve logical properties don't help one getting one's head around substructural consequence relations (an issue we will briefly touch upon in the next section), these paradoxes put pressure on Field's claim that a substructural revision of classical logic is not needed in order to adequately deal with semantic paradoxes in general. Partly for this reason, the literature on substructural approaches, both SContr-free and Cut-free, has recently been burgeoning.

On the SContr-free camp, pioneering efforts by Fitch (1942, 1948, 1950), have been followed by, among others, Mares and Paoli (2014), Shapiro (2011, 2013), Murzi and Shapiro (2014), Weber (2014), Zardini (2011, 2013c, b, 2014a, b), Meadows (2014), Caret and Weber (THIS vOLUME), and Priest (THIS volume). In particular, Zardini (2011) proves syntactic consistency for a transparent theory of truth and naïve validity whose underlying logic is a suitable strengthening of affine linear logic. Zardini (2014b) extends the theory to a SContr-free metatheory which handles the paradoxes of naïve logical properties more generally. Moreover, Zardini sketches a possible independent motivation for rejecting SContr and making sense of such a rejection. SContr is standardly invalidated on the grounds that $\phi, \phi$ and $\phi$ are different resources and hence, on the assumption that the content of a sentence is to be accounted for in terms of information, that they have different content (see e.g. Mares and Paoli 2014). Zardini (2011, 2014a) suggests instead a more conservative justification for restricting SContr, one according to which 'paradoxical' sentences are unstable, in the sense of expressing states-of-affairs which may not co-obtain with some of their consequences.

Caret and Weber (this volume), Priest (this volume), and Shapiro (THIS VOLUME) offer alternative ways of making sense of restrictions of SContr. In particular, Caret and Weber prove soundness and completeness results for a SContr-free logic for languages expressively strong enough to express their validity relation; Priest argues that the fusion connective, and its underlying structural mode of aggregating premises, intensional bunching, allows relevant logicians to provide a coherent treatment of the v-Curry Paradox; Shapiro introduces a naïve conception of sequent structure on which, he claims, SContr is naturally invalid.

On the Cut-free camp, Smiley's and Weir's pioneering efforts (Smiley 1957; Weir 2005) have been recently followed by Cobreros, Egreé, Ripley, and van Rooij, who prove model-theoretic consistency for a non-transitive, Cut-free transparent theory of truth and naïve validity (Cobreros et al. 2012, 2014). ${ }^{7}$ Ripley $(2012,2013 a$, b) has further investigated the logic and developed an inferentialist argument against Cut, a version of which is presented in Ripley (THIS vOLUME). An anti-realist nontransitive approach is presented in Tennant (2014), on the

\footnotetext{
7 The logic they advocate belongs to a family of logics that was first introduced in Zardini (2008a, b).
} 
basis of ideas first introduced in Tennant (1982, 1987, 1995, 1997).

Substructural approaches to paradox are typically consistent, although they need not be (Weber 2014). Indeed, it may be argued that they pose a threat to glut-theoretic diagnoses of the Liar and Knower paradoxes, assuming a minimal principle of uniform solution (Priest 2006b). For while the Liar and the Knower prove, according to gluttheoretic wisdom, a theorem of the form $\phi \wedge \neg \phi$, the $\mathrm{v}$-Curry Paradox allows one to 'prove' an arbitrary sentence $\phi$, and invalidating ECQ won't help avoiding triviality here. ${ }^{8}$ If the paradoxes of naïve logical properties are genuine semantic paradoxes (Beall and Murzi 2013; Murzi and Shapiro 2014; Murzi 2014a, c), then they are not uniformly solved on a standard glut-theoretic approach.

While the paradoxes of naïve logical properties seemingly show that substructural approaches are necessary to solve the semantic paradoxes via logical revision, the question naturally arises whether they are also sufficient. Zardini (2013c) contends that they are not, unless one is willing to also restrict the structural rules in one's metatheory. A certain application of Zardini's argument is criticised in Murzi (2014c). Bacon (THIS volume) argues instead that some paradoxes of identity resist substructural treatment.

\section{Revenge}

Beall (2007a) distinguishes two tasks confronting any revisionary theory of semantic paradox. The consistency project must show how languages such as English can nontrivially enjoy naïve semantic predicates, in spite of the semantic paradoxes. The point of the consistency proofs such as the ones given in Kripke (1975), Field (2008), and Zardini (2011) is precisely to prove the consistency of formal theories whose languages resemble English in relevant respects. The task is technically demanding, and by no means philosophically straightforward: for instance, the model-theoretic proofs are reassuring only insofar as the target models, which are standardly described in a classical metalanguage, adequately model the real, non-classical world. ${ }^{9}$ The expressive characterisation project, on the other hand, must indicate how it is possible, in languages such as English, to semantically characterise any sentence, as e.g. true, false, gappy, glutty, indeterminate, paradoxical, non-paradoxical, healthy, unhealthy etc. So-called contextualists (Parsons 1974a; Glanzberg 2001, 2004b, 2005) take this to be the deep root of the Liar phenomenon:

\footnotetext{
${ }^{8}$ Likewise, a natural deduction variant of the Compatibility Paradox presented in Zardini (2013c) allows one to prove $\neg \phi$, for arbitrary $\phi$ 's (Murzi 2014c).

${ }^{9}$ For more discussion, see e.g. Beall (2007b) and Bacon (2013).
}

the paradoxes arise because of a perceived need to semantically characterise 'paradoxical' sentences. In what follows, we briefly point to a possible line of argument to the effect that their diagnosis may not be far off the mark.

How, then, to characterise 'paradoxical'? Ideally, paracomplete theorists would find a property of sentences which allows them to (i) characterise 'paradoxical' sentences and (ii) justify restrictions of principles such as LEM and $\neg$-I; the paraconsistent theorists would find a property of sentences which allows them to (i) characterise 'nonparadoxical' sentences and (ii) justify the use of classical principles such as EFQ and $\neg$-E with respect to such sentences.

\subsection{Paraconsistent Revenge}

Begin with the paraconsistent case. Intuitively, in a gluttheoretic setting in which the base language- the fragment of the language which does not contain semantic predicates-is classical, non-paradoxical sentences are exactly the non-glutty sentences: the sentences that are true orfalse only. But how can such a property be expressed in the gluttheorist's language?

The fact that negation in a glut-theoretic framework fails to be exclusive implies a general difficulty to express a notion of exclusivity - exclusive truth or exclusive falsehood. It might be thought that a 'just true' predicate $\operatorname{JTr}(x)$ expressing truth only might solve the problem. But this will not do. Such a predicate would expresses the property of being true only iff the following biconditional holds: $\operatorname{JTr}(\ulcorner\phi\urcorner)$ is true iff $\phi$ really is just true. However, unsurprisingly, it is easy to see that some just true sentences are also false, as shown by a version of the Liar involving a sentence $\lambda$ identical to $\neg \operatorname{JTr}(\ulcorner\lambda\urcorner)$. One first proves by classical means $\neg \operatorname{JTr}(\ulcorner\lambda\urcorner)$, as per the standard LiarKnower recipe. But this is $\lambda$, whence via a version of the necessitation rule, $\operatorname{JTr}(\ulcorner\lambda\urcorner)$. To treat the paradox by gluttheoretic means, and accept $\operatorname{JTr}(\ulcorner\lambda\urcorner) \wedge \neg \operatorname{JTr}(\ulcorner\lambda\urcorner)$, would involve allowing for an overlap between truth and falsity only. Thus, either $\operatorname{JTr}(x)$ does not express the property of being true only after all, or, if it does, the original Liar and Knower paradoxes have not been solved.

Glut-theorists are all too well aware of the difficulty. Their standard response is that that paraconsistency 'runs deep', ${ }^{10}$ and that it should be no surprise if, in a gluttheoretic framework, just true sentences also turn out to be false (Priest 2006b; Beall 2009). Intuitively, however, one would like to be able to express that non-paradoxical sentences are precisely the consistent, non-glutty ones. And it is hard to see how such a thought can be expressed

\footnotetext{
$\overline{{ }^{10} \text { As Field }}$ (2008, p. 72) puts it in a perfectly dual context.
} 
without resorting to the classical notions of truth and falsity only.

Beall has recently proposed a different solution to the problem-one inspired by Priest (2006b, pp. 105-106). According to this, the thought that $\phi$ is just true is conveyed by the addition of a suitable shrieking rule of the form $\phi \wedge \neg \phi \vdash \perp$ to one's theory Beall (2013, 2014a). Murzi and Carrara (THIS vOLUME) raise some potential challenges for Beall's proposal; Simmons (THIS VOLUME) raises even more potential problems for paraconsistent theories. $^{11}$

\subsection{Revenge of the Knower}

Now to paracomplete, and, more generally, consistent, approaches. Here the difficulty is, dually, that we'd intuitively like to find a property $\Psi$ such that sentences such as $\kappa, \lambda$, and $\pi$ are $\neg \Psi$. Thus, in a paracomplete setting, Field identifies such a $\Psi$ with determinate truth (Field 2006, 2008); in a SContr-free setting, Zardini identifies it with stability (Zardini 2011, 2014a); in a Cut-free setting, Ripley identifies it with strict assertibility or deniability (Ripley 2013a, §\$4.1-2). However, trouble begins to arise as soon as we notice that the predicate expressing the property of being truly $\Psi$ satisfies versions of both NEC and FACT. Then, letting $\Psi$ be an operator, in each of these cases, one can define a sentence $\kappa$ identical to $\neg \Psi \operatorname{Tr}(\ulcorner\kappa\urcorner)$, and run a version of the Knower Paradox. One establishes $\neg \Psi \operatorname{Tr}(\ulcorner\kappa\urcorner)$ via a sub-argument $\Pi$ involving, by the the target theorist's lights, some illicit logical moves (one assumes $\Psi \operatorname{Tr}(\ulcorner\kappa\urcorner)$ twice and 'proves' $\neg \Psi \operatorname{Tr}(\ulcorner\kappa\urcorner)$ using inter alia $\neg$-I, SContr, and Cut). One then concludes $\Psi \operatorname{Tr}(\ulcorner\kappa\urcorner)$ via NEC and the definition of $\kappa$.

To be sure, the foregoing version of the Knower Paradox-call it the truly $\Psi$ Knower-is invalid in the target theories: after all, it is just a variant of the original Liar and Knower paradoxes, and the target theories provably solve those. However, it can be shown that standard paracomplete, SContr-free, and Cut-free theorists are still nevertheless committed to asserting $\neg \Psi \operatorname{Tr}(\ulcorner\kappa\urcorner)$ on grounds which do not themselves make use of $\neg-\mathrm{I}$, SContr, or Cut (Murzi 2014b, d). The Liar version of the foregoing argument, where $\Psi=$ 'true', is usually referred to as the Strengthened Liar Paradox (see e.g. Sainsbury 2009). We call the more general argument the Strengthened Knower.

Let us look at an example. In the paracomplete theory defended in Field (2006, 2008), $\Psi$ is a determinacy operator and the relevant $\kappa$ is equivalent to a sentence saying

\footnotetext{
11 More generally, Murzi and Carrara (THIS vOLUME) focus on whether glut-theorists are able to express disagreement, a topic also investigated in Ripley (2014). Objections to paraconsistent theories are legion; see e.g. Field (2008, Part V) and Carrara and Martino (2014).
}

that $\kappa$ is not determinately true, where $\phi$ is determinately true iff both it and $\Psi(\phi)$ are true. One can then easily generate a version of the Knower Paradox and conclude that, because of the paradox, $\kappa$ must itself be not determinately true. But this is to assert $\kappa$ itself, whence, courtesy of NEC, $\kappa$ is determinately true-contradiction. Field suggests that $\kappa$ should be deemed indeterminately determinately true instead, and that the notion of determinacy is, accordingly, intrinsically hierarchical (one can now define a sentence $\gamma$ identical to ' $\gamma$ is indeterminately determinately true', run a new version of the Knower Paradox, classify $\gamma$ as indeterminately determinately determinately true, and so on). But, one would like to argue, surely we possess a general, unified notion of indeterminacy. If so, consistency has only been restored, once more, at the price of expressive incompleteness. Field dedicates three chapters of his book Saving Truth from Paradox to the problem (Field 2008, Chaps. 22, 23, 27), arguing that there is no coherent unified notion of indeterminacy to be expressed in the first place. However, his critics are not convinced (see e.g. Simmons, THIS vOLUME and Priest 2007). ${ }^{12}$

Matters are a little more complicated in the case of SContr-free and Cut-free theories: here the truly $\Psi$ Knower doesn't immediately give grounds for asserting that $\kappa$ is itself not truly $\Psi$. All the same, in both cases it can be shown that SContr-free and Cut-free theorists are nevertheless both committed to asserting, for the relevant $\Psi$, $\neg \Psi \operatorname{Tr}(\ulcorner\kappa\urcorner)$ (Murzi 2014b, d). Contradiction now looms again, courtesy of NEC (recall, $\kappa$ and $\neg \Psi \operatorname{Tr}(\ulcorner\kappa\urcorner)$ are ex hypothesi the same sentence).

To be sure, it may be insisted that this is just a fact of life: we should have learned by now that something must be given up in light of the semantic paradoxes. Perhaps so. However, notice that the Strengthened Knower reasoning has, once again, the familiar general form of the Liar and Knower paradoxes:

\footnotetext{
12 The problem had already been identified by Kripke, who famously fell the pull towards resurrecting, because of the Strengthened Liar, 'the ghost of Tarski's hierarchy' (Kripke 1975, p. 714). For an approach that, like Field's, resorts to infinitely many notions of defectiveness, see Cook (2007). The main difference between Field and Cook, as we see it, is that, while Field rejects (implausibly, in our view) the existence of a unified notion of indeterminacy, or, more generally, defectiveness, following Dummett (1991), Cook rejects absolute generality instead - the possibility of quantifying over absolutely everything. As a result, one cannot quantify over absolutely all the semantic categories other than true, and would-be revenge sentences such as $\kappa=\kappa$ falls in one of the semantic categories other than true actually don't breed revenge. Our main concern about Cook's strategy is that (as in Field's case) it solves the Liar and the Strengthened Liar in different ways. Yet, as we argue below, there are reasons for thinking that they are essentially the same paradox and that, for this reason, they should receive a uniform treatment.
} 


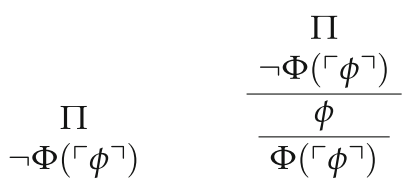

The only difference is that, in the case of the Strengthened Knower, $\Pi$ is no longer a subproof from two occurrences of $\Psi \operatorname{Tr}(\ulcorner\kappa\urcorner))$ to $\perp$ involving uses of $\neg-\mathrm{I}$, SContr, and Cut. Thus, it might be thought, one cannot in general solve the Strengthened Knower by blaming such rules. But if the Liar Paradox, the Knower Paradox, and the Strengthened Knower are all essentially the same paradox, in virtue of sharing the same general form, it is tempting to conclude that one cannot in general solve the semantic paradoxes by weakening the logic. Or so one might argue. ${ }^{13}$

Acknowledgments Most of the articles contained in this volume were presented at the conference Paradox and Logical Revision, held in Munich at the Munich Center for Mathematical Philosophy, Ludwig-Maximilians-Universität, in July 2012. The conference was organised by the editors of the present volume and Ole T. Hjortland, and was generously funded by the German Research Foundation (DFG) and the Alexander von Humboldt Foundation. The first author is grateful to the British Academy, the Alexander von Humboldt Foundation, and the School of European Culture and languages at the University of Kent for generous financial support during the time this paper was written.

\section{References}

Asenjo FG (1966) A calculus of antinomies. Notre Dame J Form Log 16:103-105

Asenjo F, Tamburino J (1975) Logic of antinomies. Notre Dame J Form Log 16:17-44

Bacon A (2013) Non-classical metatheory for non-classical logics. J Philos Log 42(2):335-355

Bacon A (2014) Indeterminacy and Revenge. Philos Rev 88:590-618

Beall J (2007a) Truth and paradox: a philosophical sketch. In: Jacquette D (ed) Philosophy of logic. Elsevier, Oxford, pp 325-410

Beall J (ed) (2007b) Revenge of the liar. Oxford University Press, Oxford

Beall J (2009) Spandrels of truth. Oxford University Press, Oxford

Beall J (2011) Multiple-conclusion LP and default classicality. Rev Symb Log 4(1):326-336

Beall J (2013) Shrieking against gluts: the solution to the 'just true' problem. Analysis 73(3):438-45

Beall J (2014a) A simple approach towards recapturing consistent theories in paraconsistent settings. Rev Symb Log (Forthcoming)

Beall J (2014b) Truth without detachment. MS

Beall J, Murzi J (2013) Two flavors of Curry's paradox. J Philos CX(3):143-165

\footnotetext{
13 Priest (2006a, Ch. 1; 2007) and Scharp (2013, Ch. 4) both describe a similar revenge argument. For a general revenge argument against certain (widespread) classical approaches to semantic paradox, see Bacon (2014).
}

Beall J, Ripley D (2014) Non-classical theories of truth. In: Glanzberg $M$ (ed) Oxford Handbook of Truth (Forthcoming)

Beall JC, Brady R, Hazen AP, Priest G, Restall G (2006) Relevant restricted quantification. J Philos Logic 35:587-598

Brady R (2006) Universal logic. CSLI Publications, Stanford

Burge T (1979) Semantical paradox. J Philos 76(4):169-198

Carrara M, Martino E (2014) Logical consequence and conditionals from a dialetheic perspective. Log Anal 227:359-378

Cobreros P, Egré P, Ripley D, van Rooij R (2012) Tolerant, classical, strict. J Philos Log 41(2):347-385

Cobreros P, Égré P, Ripley D, van Rooij R (2014) Reaching transparent truth. Mind (Forthcoming)

Cook R (2007) Embracing revenge: on the indefinite extensibility of language. In: Beall (ed) Revenge of the Liar: New Essays on the Paradox. Oxford University Press, pp 31-52

Cook R (2013) There is no paradox of logical validity. Log Univers (Forthcoming)

Corcoran J (ed) (1983) Logic, semantics, metamathematics, 2nd edn. Hackett Publishing Company, Indianapolis

Dummett (1991) Frege: Philosophy of Mathematics, Harvard University Press

Field H (2006) Truth and the unprovability of consistency. Mind 115(459):567-606

Field H (2008) Saving truth from paradox. Oxford University Press, Oxford

Field H (2013) Naive truth and restricted quantification: saving truth a whole lot better. Rev Symb Log 7(1):147-191

Field H (2014) Disarming a paradox of validity. Notre Dame J Form Log (Forthcoming)

Fitch F (1942) A basic logic. J Symb Log 7:105-114

Fitch F (1948) An extension of basic logic. J Symb Log 13:95-106

Fitch F (1950) A further consistent extension of basic logic. J Symb Log 14:209-218

Glanzberg M (2001) The liar in context. Philos Stud 103(3):217-251

Glanzberg M (2004a) A contextual-hierarchical approach to truth and the liar paradox. J Philos Log 33:27-88

Glanzberg M (2004b) Quantification and realism. Philos Phenomenol Res 69(3):541-572

Glanzberg M (2005) Truth, reflection, and hierarchies. Synthese 142(3):289-315

Goodship L (1996) On dialetheism. Australas J Philos 74:153-161

Gupta A, Belnap N (1993) The revision theory of truth. MIT Press, Cambridge

Horsten L (2009) Levity. Mind 118(471):555-581

Horsten H (2011) The Tarskian turn. MIT Press, Cambridge

Kaplan D, Montague R (1960) A paradox regained. Notre Dame J Form Log 1:79-90

Ketland J (2012) Validity as a primitive. Analysis 72(3):421-430

Kripke S (1975) Outline of a theory of truth. J Philos 72:690-716

Leitgeb H (2005) What truth depends on. J Philos Log 34(2):155-192

Mares E, Paoli F (2014) Logical consequence and the paradoxes. J Philos Log 43:439-469

Martin DA (2011) Field's saving truth from paradox: some things it doesn't do. Rev Symb Log 4(3):339-347

Maudlin T (2004) Truth and paradox: solving the riddles. Oxford University Press, New York

McGee V (1991) Truth, vagueness and paradox. Hackett, Indianapolis

Meadows T (2014) Fixed-points for consequence relations. Log Anal (Forthcoming)

Murzi J (2012) On Heck's new liar. Thought 1(4):258-69

Murzi J (2014a) The inexpressibility of validity. Analysis 74(1):65-81

Murzi J (2014b) Instability and revenge. Unpublished manuscript

Murzi J (2014c) Naïve logical properties and higher-order reasoning. Unpublished manuscript

Murzi J (2014d) Saving logic from paradox. Unpublished manuscript 
Murzi J, Shapiro L (2014) Validity and truth-preservation. In: Achourioti HGT, Fujimoto F, Martinez-Fernandez J (eds) Unifying the philosophy of truth. Springer, Berlin (Forthcoming)

Myhill J (1960) Some remarks on the notion of proof. J Philos 57(14):461-471

Negri S, von Plato J (2001) Structural proof theory. Cambridge University Press, Cambridge

Parsons C (1974a) The liar paradox. J Philos Log 3(4):381-412

Parsons C (1974b) Sets and classes. Noûs 8:1-12

Priest G (1979) The logic of paradox. J Philos Log 8:219-241

Priest (2006a) In contradiction

Priest G (2006b) Doubt truth to be a liar. Oxford University Press, Oxford

Priest G (2007) Revenge, field, and ZF. In: Beall J (ed) Revenge of the liar. Oxford University Press, Oxford, pp 225-233

Ripley D (2012) Conservatively extending classical logic with transparent truth. Rev Symb Log 5:354-378

Ripley D (2013a) Paradoxes and failures of cut. Australas J Philos 91(1):139-164

Ripley D (2013b) Revising up. Philosophers' Imprint 13(5):1-13

Ripley D (2014) Embedding denial. In: Caret C, Hjortland OT (eds) Foundations of logical consequence. Oxford University Press, Oxford (Forthcoming)

Sainsbury M (2009) Paradoxes, 3rd edn. Cambridge University Press, Cambridge

Scharp K (2013) Replacing truth. Oxford University Press, Oxford

Schurz C (2011) Contextual apporaches to truth and the strengthened liar paradox. $\mathrm{PhD}$ thesis, University of Salzburg

Shapiro L (2011) Deflating logical consequence. Philos Q 61:320-342

Shapiro L (2013) Validity curry strengthened. Thought 3:100-107

Simmons K (1993) Universlity and the liar: an essay on truth and the diagonal argument. Cambridge University Press, Cambridge

Simmons K (2000) Sets, classes and extensions. A singularity approach to Russell's paradox. Philos Stud 100(2):109-149

Smiley T (1957) Entailment and deducibility. Proc Aristot Soc 59:233-254

Soames S (1999) Understanding truth. Oxford University Press, Oxford
Tarski A (1936) O pojciu wynikania logicznego. Prezglad Filozoficzny 39:97-112 (Translated as "On the Concept of Logical Consequence" in Tarski (1956), pp. 409-420)

Tennant N (1982) Proof and paradox. Dialectica 36:265-96

Tennant N (1987) Anti-realism and logic. Clarendon Press, Oxford

Tennant N (1995) On paradox without self-reference. Analysis 55:99-207

Tennant N (1997) The taming of the true. Oxford University Press, Oxford

Tennant N (2014) A new unified account of truth and paradox. Mind (Forthcoming)

Weber Z (2014) Naïve validity. Philos Q 64(254):99-114

Weir A (2005) Naïve truth and sophisticated logic. In: Beall J, Armour-Garb B (eds) Deflationism and paradox. Oxford University Press, Oxford, pp 218-249

Whittle B (2004) Dialetheism, logical consequence and hierarchy. Analysis 64(4):318-26

Williamson T (1998) Indefinite extensibility. Grazer Philos Stud $55: 1-24$

Zardini E (2008a) Living on the slippery slope: the nature, sources and logic of vagueness, $\mathrm{PhD}$ thesis, University of St Andrews

Zardini E (2008b) A model of tolerance. Stud Log 90(3):337-368

Zardini E (2011) Truth without contra(di)ction. Rev Symb Log $4: 498-535$

Zardini E (2013a) It is not the case that [ $p$ and 'it is not the case that $p$ ' is true] nor is it the case that [ $p$ and ' $p$ ' is not true]. Thought 1(4):309-319

Zardini E (2013b) Naïve modus ponens. J Philos Log 42(4):575-593

Zardini E (2013c) Näive logical properties and structural properties. J Philos 110(11):633-644

Zardini E (2014a) Getting one for two, or the contractors' bad deal. Towards a unified solution to the semantic paradoxes. In: Achourioti HGT, Fujimoto F, Martinez-Fernandez J (eds) Unifying the philosophy of truth. Springer, Berlin (Forthcoming)

Zardini E (2014b) Naïve truth and naïve logical properties. Rev Symb $\log 7(2): 351-384$

Zardini (2014c) A and Omega, Unpublished manuscript 